\title{
Capacidad de identificar mamíferos nativos y exóticos entre los habitantes de San Ramón, Costa Rica: papel de edad, sexo, educación y origen (rural o urbano)
}

\author{
Melvin Cartín Núñez ${ }^{1 *}$, Verónica Sánchez Acuña ${ }^{2}$, Gladis Abarca Segura² \\ 1. Universidad de Costa Rica, Sede de Occidente, Sección de Biología; melvin.cartin@ucr.ac.cr \\ 2. Universidad de Costa Rica, Sede de Occidente, Gestión de Recursos Naturales; veronik2418@gmail.com; gladisa90@gmail.com
}

Recibido 27-IV-2015 • Corregido 23-VI-2015 • Aceptado 10-VII-2015

\begin{abstract}
Ability to identify native and exotic mammals, among the inhabitants of San Ramon, Costa Rica: role of age, sex, education and origin (rural or urban). Costa Rica is a country with a high biological diversity that protects one quarter of its territory and has the highest educational level in Central America. We expected Costa Ricans to be familiar with their mammals. To test our hypothesis, we asked a sample of citizens of San Ramon, Costa Rica, to identify photographs of mammals, both native and exotic. Against our hypothesis, they identified less native than exotic species. Men recognized more native species than women, and the youngest persons more exotic species. Education and rural or urban origin made no difference. Mass media play a key role in the transmission of knowledge about biodiversity; this explains why exotic species (which have greater coverage in the media) are better known than some local species.
\end{abstract}

Key words: local knowledge, mammals, local species, exotic species, biodiversity, Costa Rica.

Costa Rica es reconocida a nivel internacional como una de las naciones de mayor diversidad biológica. Según datos del Instituto Nacional de Biodiversidad (INBio), en el país se encuentran más de medio millón de especies, lo cual representa casi el $4 \%$ del total de especies en el planeta (INBio, 2013). Esta gran diversidad es uno de los mayores atractivos para los turistas extranjeros que visitan el país.

De igual forma, Costa Rica es reconocida por sus esfuerzos en materia de conservación (Campbell, 2002; Salazar, 2009; Hoffman, 2011). Una cuarta parte del territorio continental se encuentra bajo alguna categoría de protección, por ejemplo, Parques Nacionales,
RESUMEN: Costa Rica es un país con alta diversidad biológica y mantiene una cuarta parte de su territorio bajo alguna categoría de protección. Por ello, se podría esperar que los costarricenses conozcan y aprecien la biodiversidad local. Por lo que a una muestra de ciudadanos del cantón de San Ramón, provincia de Alajuela, les solicitamos identificar una serie de fotografías de mamíferos, nativos y exóticos. Encontramos que el número de especies nativas identificadas fue menor que el número de especies exóticas. Los hombres reconocieron más especies nativas que las mujeres. Las personas más jóvenes identificaron más especies exóticas, pero no hallamos diferencias significativas en el número de especies identificadas según el origen de la persona (rural o urbano), o el nivel de escolaridad. Los medios de comunicación masiva, como la televisión o el internet, juegan un papel fundamental en la transmisión de conocimientos sobre la biodiversidad, lo que explica porqué las especies exóticas, con mayor cobertura y difusión que especies locales, son mejor conocidas.

Palabras clave: conocimiento local, mamíferos, especies nativas, especies exóticas, biodiversidad, Costa Rica.
Reservas Biológicas, Refugios de Vida Silvestre y Reservas Forestales son parte de las diferentes categorías de manejo dedicadas a la protección de recursos naturales. Además, existe una red de Reservas Naturales Privadas que coadyuva en los esfuerzos de conservación hechos por el país (Cartín, 2010; Bagley \& Johnson, 2014).

Otro de los logros de la nación costarricense es su nivel educativo. El nivel de alfabetismo supera el $96 \%$, lo cual la ubica entre las más altas del mundo y la primera en América Latina (Costa Rica Country Profile, 2012; Coalición Costarricense de Iniciativas de Desarrollo [CINDE], 2012; Sada, 2015). Existe una buena cobertura a nivel de educación primaria, secundaria, técnica y 
universitaria que ha generado beneficios al país. Según González (2011) para el año 2011, más de 200 empresas multinacionales se habían establecido en Costa Rica, gracias a la buena preparación del recurso humano en áreas técnicas y especializadas, así como en el dominio de una segunda lengua, principalmente el inglés (AguilarSánchez, 2005). Para el año 2014, la inversión extranjera directa se estimó en 2,2 miles de millones de dólares norteamericanos (Sada, 2015).

Siendo Costa Rica un país con un buen nivel educativo, con una gran riqueza biológica y con un reconocido compromiso en materia ambiental, sería de esperar que el costarricense fuese una persona que muestre comportamientos y actitudes tendientes a valorar la biodiversidad, es decir, una persona bioalfabetizada. No obstante, cuencas contaminadas, ciudades sucias, botaderos de basura a cielo abierto, deforestación y otros problemas parecen ser el reflejo de actitudes en la dirección contraria. Incluso en aspectos más sencillos, como por ejemplo, el grado de conocimiento de las especies locales, es probable que no se obtenga una buena nota.

¿Cuántas especies nativas somos capaces de identificar? Es imposible esperar que alguien llegue a conocer y reconocer cada una de las 500 mil o más especies presentes en el país, pero lo ideal sería que sí pudiese reconocer aquellas especies más conspicuas o más familiares. No obstante, la experiencia de los autores hace pensar que la población costarricense conoce muy poco sobre la fauna silvestre aun presente en los diferentes ecosistemas de Costa Rica.

Para poner a prueba lo anterior, nos propusimos explorar cuál es el conocimiento de las especies locales que tiene la población costarricense. Enfocamos nuestro esfuerzo en un grupo particular, el de los mamíferos, por ser esta clase de animales a la que pertenece también nuestra especie y por ser el grupo de organismos con el cual, probablemente, hemos establecido mayor empatía (por ejemplo a través del uso de mascotas, animales de granja o de trabajo). Para efectos de comparación, también quisimos poner a prueba cuánto conoce la población sobre especies de mamíferos exóticas, pues creíamos que la gente identificaría más especies exóticas que locales.

\section{MÉTODOS}

Los datos los recolectamos en el cantón de San Ramón, provincia de Alajuela. Dicho cantón está localizado en el occidente de la región central de Costa Rica y su principal centro urbano está próximo al límite occidental del Gran Área Metropolitana (GAM) (Rosales, 2012). Las razones para elegir San Ramón fueron las siguientes: i) posee tanto población urbana como rural ii) posee instituciones educativas, públicas y privadas, en todos los niveles (preescolar, primaria, secundaria, técnica, universitaria) iii) existe amplia variedad de actividades comerciales y iv) cerca del 50\% de su territorio está cubierto de bosque (Araúz-Almengor, Soto Castro, Vargas Bolaños, Arias-Ramírez y Nuñez Corrales, 2007), por lo que aún es posible encontrar gran cantidad de fauna nativa."

El trabajo de campo lo realizamos entre los meses de octubre y noviembre de 2012. Para ello elaboramos un cuestionario que se aplicó a una muestra de 100 personas mayores de edad, elegidas al azar en el parque central de la ciudad, feria del agricultor y terminal de autobuses distritales. A cada uno se le preguntó por el lugar de residencia, la edad, la escolaridad y el sexo. Asimismo se les presentó una serie de 10 fotografías de mamíferos

\section{CUADRO 1}

Lista de especies utilizadas en el estudio

\section{Especies nativas}

Cabro de monte (Mazama americana Erxleben, 1777)

Chancho de monte (Tayassu pecari Link, 1795)

Danta (Tapirus bairdii Gill, 1895)

Guatusa (Dasyprocta punctata Gray, 1842)

Jaguar (Panthera onca Linnaeus, 1758)

Oso hormiguero (Tamandua mexicana Saussure, 1860)

Puma (Puma concolor Linnaeus, 1771)

Tepescuintle (Cuniculus paca Linnaeus, 1766)

Tolomuco (Eira barbara Linnaeus, 1758)

Zorro cuatro ojos (Philander opossum Linnaeus, 1758)

\section{Especies exóticas}

Canguro (Macropus rufus Desmarest, 1822)

Cebra (Equus quagga Boddaert, 1785)

Elefante (Loxodanta africana Blumenbach, 1797)

Hiena (Crocuta crocuta Erxleben, 1777)

Hipopótamo (Choeropsis liberiensis Morton, 1849)

Jabalí (Phacochoerus africanus Gmelin, 1788)

Jirafa (Giraffa camelopardalis Linnaeus, 1758)

León (Panthera leo Linnaeus, 1758)

Reno (Rangifer tarandus Linnaeus, 1758)

Tigre (Panthera tigris Linnaeus, 1758)

Nombres comunes según Valerio (1978) 
terrestres nativos y 10 de mamíferos terrestres exóticos para su identificación (cuadro 1).

Tratamiento de los datos: Para determinar la relación entre el número de especies identificadas correctamente y cada una de las variables medidas (edad, procedencia y escolaridad) el nivel de escolaridad se clasificó en ocho opciones: ninguna, primaria incompleta, primaria completa, secundaria incompleta, secundaria completa, parauniversitaria o técnica, universitaria incompleta y universitaria completa.

Análisis estadístico: Para determinar si existían diferencias en el porcentaje de identificaciones correctas entre especies exóticas y nativas así como la relación entre las distintas variables y el porcentaje de especies nativas identificadas, se llevó a cabo un análisis de varianza de mediciones repetidas, con el programa informático Systat $10^{\oplus}$.

\section{RESULTADOS}

Caracterización de la población: La categoría con mayor número de personas en la muestra fue la de 18

CUADRO 2

Distribución de frecuencias según sexo y edad de los participantes

\begin{tabular}{lccc}
\multicolumn{1}{c}{ Categoría de edad } & Hombres & Mujeres & Total \\
18 a 29 años & 16 & 22 & 38 \\
30 a 39 años & 7 & 7 & 14 \\
40 a 49 años & 9 & 8 & 17 \\
50 a 59 años & 9 & 5 & 14 \\
60 años o más & 14 & 3 & 17 \\
Total & $\mathbf{5 5}$ & $\mathbf{4 5}$ & $\mathbf{1 0 0}$ \\
\hline
\end{tabular}

a 29 años y la de menor número fue la de 30 a 39 años (Cuadro 2). En términos generales, la proporción de sexos dentro de la muestra (hombre: mujer) fue de 1:1, excepto en las categorías de 50 a 59 años y 60 años o más. En esta última se registró la relación de género más desigual, casi de 4:1 (Cuadro 2).

Conocimiento sobre mamíferos terrestres: El número de especies identificadas correctamente fue diferente para mamíferos exóticos y mamíferos nativos $(F=272,659 \mathrm{gl}=1 \mathrm{p}<0,001$ ) (figura 1 ). Las personas, en promedio, identificaron mayor número de especies exóticas $(8,25 \pm 1,62)$ que especies nativas, cuyo número correctamente identificado no llega ni a la mitad de las especies mostradas $(4,39 \pm 2,18)$. No se encontraron diferencias en el porcentaje de identificaciones correctas según el lugar de residencia de las personas encuestadas $(F=0,062 \mathrm{gl}=1$ $\mathrm{p}=0,803)$ pero sí al considerar el sexo del individuo ( $\mathrm{F}=$ $4,324 \mathrm{gl}=1 \mathrm{p}=0,041$ ) y el grupo de edad al que pertenecía $(F=3,899 \mathrm{gl}=4 \mathrm{p}=0,006)$. Específicamente, se encontró que los hombres identifican un mayor número de especies nativas que las mujeres (figura 2) y el porcentaje de aciertos en la identificación de especies exóticas disminuye conforme aumenta la edad de los entrevistados (figura 3). Con respecto a la escolaridad, no se hallaron diferencias en el número de identificaciones correctas según el nivel académico ( $F=1,069 \mathrm{gl}=7 \mathrm{p}=0,391)$.

\section{DISCUSIÓN}

La población estudiada parece conocer mejor las especies de mamíferos foráneas que las autóctonas. Este resultado probablemente se explica, al menos en parte, por la influencia de los medios de comunicación, en particular la televisión. Es bastante común observar diferentes tipos de audiovisuales (películas, documentales,

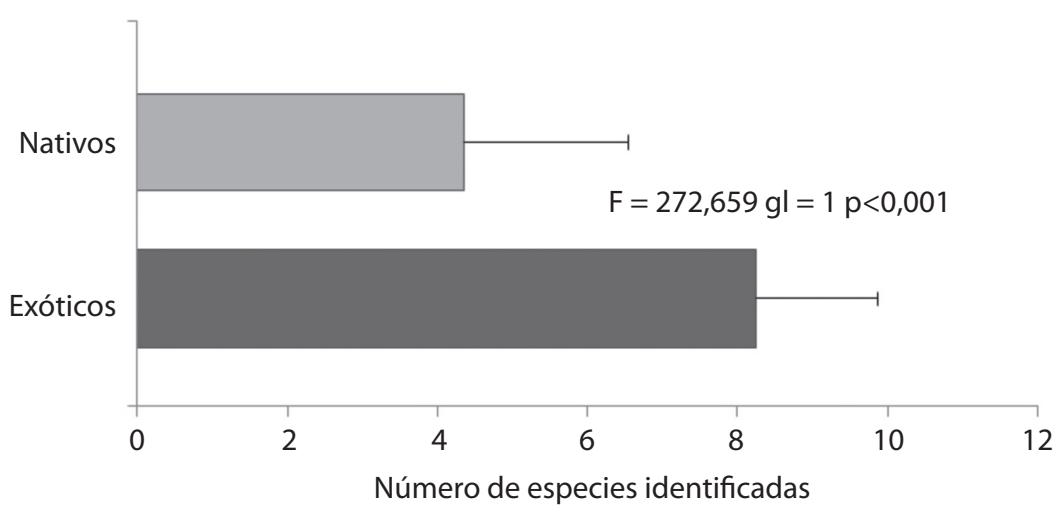

Fig. 1. Mamíferos nativos versus exóticos. Identificación exitosa de especies. 


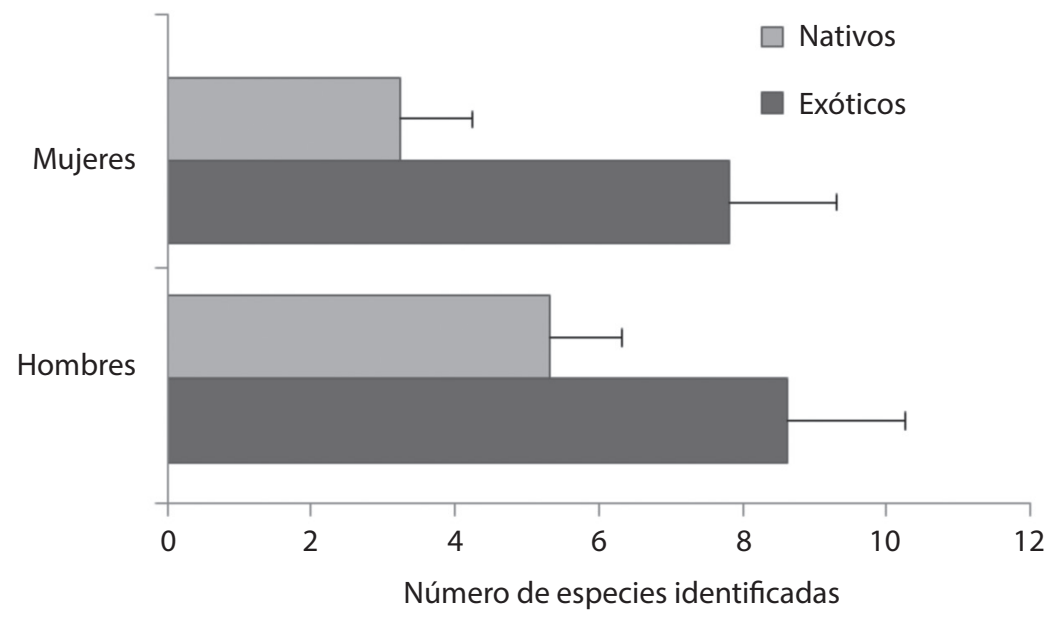

Fig. 2. Mamíferos nativos versus exóticos. Identificación exitosa de las especies según el sexo de los participantes.

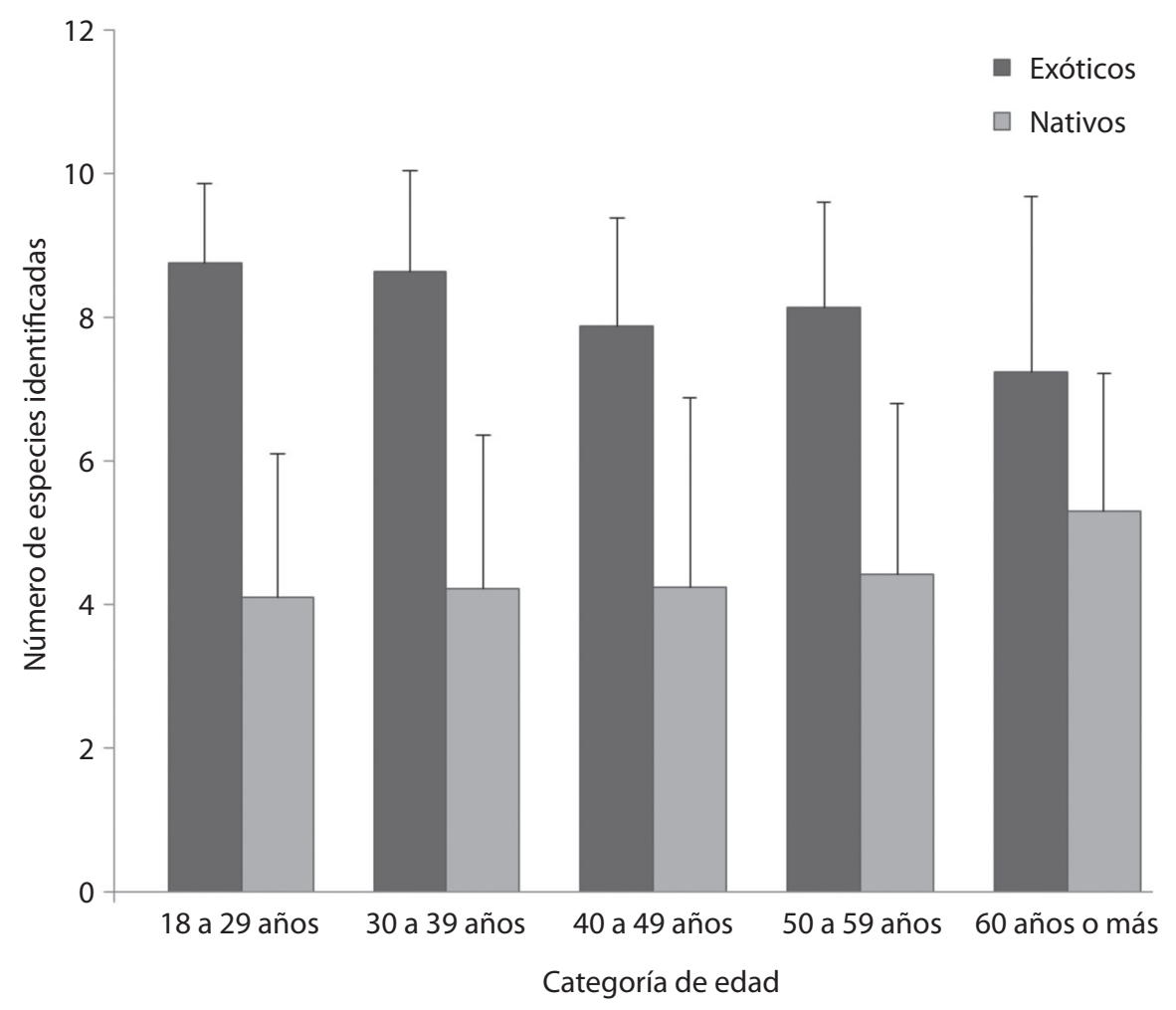

Fig. 3. Mamíferos nativos versus exóticos. Identificación exitosa de las especies según la edad de los entrevistados.

cortometrajes, entre otros) dedicados a difundir información sobre la fauna de otras regiones pero no así de la fauna local. En especial sobresale el caso de las especies africanas. Desde la llegada de las cámaras que acompañaban a los exploradores y cazadores europeos, la mastofauna africana se convirtió en blanco de entretenimiento para la población occidental (ver Beinart \&
McKeon, 2009). Muchos de los espacios televisivos son cubiertos con producciones cuyo centro de atención son estos animales.

Gracias a esta difusión masiva, especies como las jirafas, los elefantes, los chimpancés y los leones, entre otras, son reconocidas por la mayoría de la población. De igual forma, la imagen de estos animales ha sido explotada 
comercialmente en productos de todo tipo como juguetes, peluches, artesanía, ropa y otros accesorios dirigidos principalmente a la población infantil. Esta actividad comercial ha ido de la mano con la industria de los dibujos animados $y$, en épocas más recientes, la animación digital. De hecho, muchas de las personas cuando se refieren a un animal lo hacen por el nombre de algún personaje animado y no propiamente por el nombre común de la especie (e.g. "Bambi" para referirse a un venado, o "Dumbo" para referirse a un elefante-Bambi y Dumbo son los nombres de dos personajes de películas animadas de los Estudios Walt Disney ${ }^{{ }^{-}}$).

Asimismo, gran cantidad de empresas transnacionales utilizan animales exóticos como logo para su marca. Especies como el puma, el tigre, el oso polar, el oso panda, la jirafa y el león son asociados por los consumidores casi de forma automática con diferentes marcas de calzado deportivo, cereales, refrescos gaseosos, juguetes y productoras cinematográficas, respectivamente. Lo mismo ocurre para entidades comerciales propias de Costa Rica. Por ejemplo si nos fijamos en la industria del fútbol (el deporte más seguido por los costarricenses), de los cuatro equipos con mayor afición, solo uno, Cartaginés, tiene como mascota oficial un animal nativo, el manigordo. En cambio, Alajuelense, Saprissa y Herediano usan como imagen un león, un "monstruo" (similar a un dragón) y un tigre respectivamente. Esto es una muestra de cómo se pierde la oportunidad de aprovechar el atractivo de cualquier organismo perteneciente a la gran diversidad biológica presente en el país.

En el año 2013, con motivo de los X Juegos Deportivos Centroamericanos cuya sede fue Costa Rica, el comité organizador eligió al jaguar "Namú" como la mascota oficial de dicho evento (Leandro, 2012) (figura 4). Si bien la elección del jaguar se puede destacar dentro de los esfuerzos por afianzar una identidad nacional con

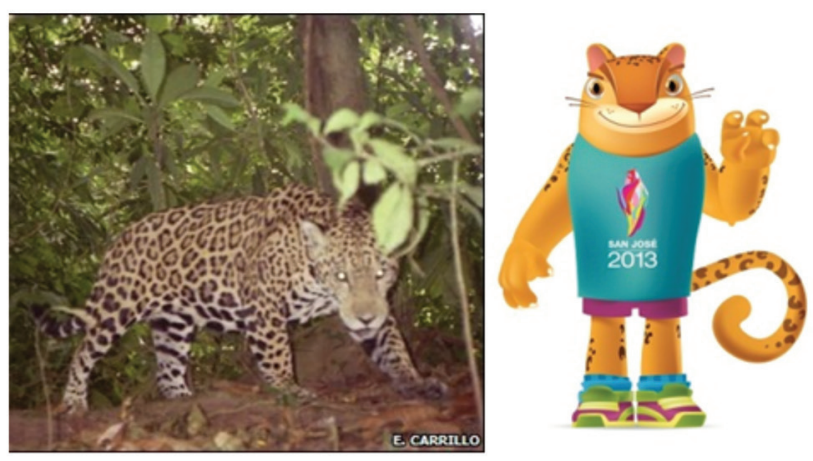

Fig. 4. Namú, la mascota oficial de los X Juegos Deportivos Centroamericanos, San José 2013. (Jaguar: Fotografía de Eduardo Carrillo; Namú: Periódico La Nación) elementos propios de la biodiversidad local, una de las críticas hechas en su momento consistió en que la imagen de Namú no guarda mucha similitud con un jaguar real por lo que podría ser difícil asociarlo con la especie a la cual representa. Eso sí, reiteramos, vale la pena resaltar la elección de Namú como mascota, pues es uno de los pocos ejemplos en los que se ha logrado promocionar elementos de la biodiversidad local a través de actividades no necesariamente relacionadas con la conservación de la naturaleza, como en este caso un evento deportivo a nivel regional.

Por otra parte, uno de los resultados más llamativos, es el no haber encontrado diferencias en el número de identificaciones correctas entre la población rural y la población urbana. En principio creíamos que las personas de zonas rurales identificarían mayor número de especies nativas, pues en teoría, se tiene una mayor probabilidad de ver mamíferos silvestres en el campo con respecto a la ciudad y con ello también la probabilidad de saber de cuál especie se trata. No obstante, nuestros resultados sugieren una homogenización del conocimiento de la fauna local, por desgracia muy pobre.

¿Qué razones explican este resultado? Una de ellas podría ser que debido al aumento de la urbanización y la expansión de la frontera agrícola, cada vez es más difícil observar mamíferos silvestres. Si bien algunos organismos pueden tomar ventaja de la variedad de fuentes alimenticias en áreas urbanizadas (como por ejemplo insectos, árboles frutales, basura, incluso mascotas) (Riley, 2006), también es cierto que se ven más expuestos a la cacería intencional o accidental y con ello se reducirían sus poblaciones o podría variar su comportamiento. Además, a excepción del tolomuco y de la guatusa, las especies locales cuyas imágenes se utilizaron en el cuestionario, son sensibles a la fragmentación del bosque, por lo que incluso en zonas rurales sus avistamientos podrían no ser tan comunes.

Con respecto a género, los varones logran reconocer más especies nativas que las mujeres, aun y cuando ambos sexos reconocen por igual las especies exóticas. Al revisar los datos, se nota que las especies nativas más identificadas fueron aquellas relacionadas con la cacería (e.g. tepescuintle, chancho de monte, guatusa), hábito meramente masculino (Sánchez, 2006). En cambio, ver la televisión o navegar en internet no necesariamente es un hábito exclusivo de algún sexo y es a través de estos medios que probablemente se recibe la mayor cantidad de información sobre especies exóticas.

Al analizar la variable de la edad, los grupos de personas de menor edad reconocen más especies exóticas que los mayores pero la situación se invierte ligeramente 
cuando se trata de especies nativas. Es factible suponer que este resultado se deba a una mayor influencia de los medios de comunicación y la publicidad comercial en los niños y jóvenes del país, tal y como sucede en otras partes del mundo (Huxham, Welsh, Berry \& Templeton, 2006; Ballouard, Brischoux \& Bonnet, 2011). Por su parte, las personas de mayor edad, aquellos de cincuenta años en adelante, quizás tuvieron la oportunidad de crecer en un entorno menos deteriorado y de esta forma pudieron haber tenido (más) contacto con especies nativas de Costa Rica.

Finalmente, el hecho de que no haya diferencias en la identificación de especies de acuerdo con la escolaridad, indica que el conocimiento de las especies no se relaciona con el nivel de educación formal de los habitantes. En principio uno podría esperar que la probabilidad de haber recibido algún tipo de educación ambiental, haya sido mayor en las personas que han estado dentro del sistema educativo, con respecto a las que no, y que aumente conforme se asciende en los niveles escolares. Sin embargo, la educación ambiental no se ha logrado implementar de manera satisfactoria en los sistemas educativos, a pesar de que fue uno de los temas principales en la Cumbre de la Tierra de Estocolmo (Kassas, 2002).

Aunque el país ha hecho esfuerzos en materia de educación ambiental, el conocimiento de la biodiversidad local no forma parte de los objetivos en la mayoría de programas. La atención está dirigida al desarrollo de conciencia y sensibilidad en relación con el uso, cuidado y preservación del medio ambiente (Solano, 2006). Como ejemplo de lo que sucede en la práctica, en un estudio realizado por Benavides (2008) en diferentes escuelas de la región de San Carlos, la autora encontró que el aprendizaje ambiental se suscribe principalmente a temas de limpieza urbana, aseo del centro educativo y acciones en ahorro de energía.

A manera de epílogo, encontramos que la población en general reconoce más especies de mamíferos exóticas que locales, resultado probablemente influenciado por la divulgación diferenciada de información sobre ambos grupos en los distintos medios de comunicación como por ejemplo la televisión. Además, fueron los varones quienes reconocieron más especies nativas que las mujeres, aunque no hubo diferencias con respecto a las especies foráneas. Las diferencias en la identificación de las especies locales según el género, podrían tener alguna relación con las representaciones sociales de la población, donde hábitos que involucran el conocimiento de la fauna, como la cacería por ejemplo, se asocian con el sexo masculino y no con el femenino.

Las personas más jóvenes reconocen más especies exóticas que las mayores. Presumiblemente los jóvenes tienen más influencia de los medios de comunicación, en especial la televisión y el internet. Estos medios en general han contribuido a que los usuarios tiendan a conocer relativamente bien a unas pocas especies de mamíferos icónicas a nivel mundial, fenómeno estudiado y reportado por Ballouard et al. (2011) en diferentes naciones de diferentes continentes. Tal parece que Costa Rica ha experimentado este mismo fenómeno.

Finalmente, el pobre conocimiento de las especies locales en comparación con el de las especies exóticas, no depende del nivel de escolaridad ni del lugar de residencia de las personas. Esto nos podría indicar un par de debilidades a nivel de país: primero, que el conocimiento de la biodiversidad local no forma parte de las prioridades en los distintos programas de educación ambiental del sistema educativo formal o informal y segundo, que la pérdida de diversidad local avanza a un ritmo tan acelerado que incluso impide que las personas, residentes en lugares donde se esperaría mayor contacto con las especies silvestres, lleguen a conocerlas.

\section{REFERENCIAS}

Aguilar-Sánchez, J. (2005). English in Costa Rica. World Englishes, $24(2), 161-172$.

Araúz-Almengor, M.; Soto Castro, C.; Vargas Bolaños, Ch.; AriasRamírez, L. y S. Nuñez Corrales. (2007). Cobertura boscosa y otros usos del suelo del cantón de San Ramón, Costa Rica: Análisis de los bloques boscosos. En: Congreso de Geoprocesamiento. San José, Costa Rica. Universidad Estatal a Distancia. Recuperado de: http://www.cenat.ac.cr/CongresoGeoprocesamiento/contenidos/ ponencias/MArauz_et_al.pdf.

Bagley, J. \& Johnson, J. (2014). Phylogeography and biogeography of the lower Central American Neotropics: diversification between two continents and between two seas. Biological Reviews, 89, 767-790.

Ballouard, J-M., Brischoux, F. \& X. Bonnet. (2011). Children prioritize virtual exotic biodiversity over local biodiversity. PLOS ONE, 6 (8), 1-8.

Beinart W. \& McKeon, K. (2009). Wildlife media and representations of Africa, 1950s to the1970s. Environmental History, $14,429-452$.

Benavides, L. (2008). Impacto de la cultura escolar y la práctica pedagógica en el aprendizaje ambiental en las escuelas técnicas del circuito escolar 03 de la Dirección Regional en Educación de San Carlos, Costa Rica. (Tesis de Doctorado). Universidad Nacional, Universidad Estatal a Distancia, Instituto Tecnológico de Costa Rica.

Campbell, I. (2002). Conservation narratives in Costa Rica: Conflict and co-existence. Development and Change, 33, 29-56.

Cartín, M. (2010). Estimación de riqueza, composición y abundancia relativa de mamíferos terrestres en dos zonas 
protegidas en Costa Rica con miras a su sostenibilidad. (Tesis de maestría). Universidad de Costa Rica. San Ramón, Costa Rica.

Coalición Costarricense de Iniciativas de Desarrollo (CINDE). (2012). Educación en Costa Rica. San José, Costa Rica. CINDE.

Costa Rica Country Profile. (2012). Current Politics and Economics of South and Central America, 5 (3), 387-398. Recuperado de: http://search.proquest.com/docview/1707984923? accountid $=28692$

González, A. (2011, mayo 27). La atracción de empresas extranjeras a Costa Rica. En: La Nación. Foro de Opinión. Recuperado de: http://www.nacion.com/2011-05-27/ Opinion/Foro/Opinion2791572.aspx

Hoffman, D. (2011). Do global statistics represent local reality and should they guide conservation policy?: Examples from Costa Rica. Conservation and Society, 9 (1), 16-24.

Huxham, M., Welsh, A., Berry, A. \& Templeton, S. (2006). Factors influencing primary school children's knowledge of wildlife. Journal of Biological Education, 4 (1), 9-12.

Instituto Nacional de Biodiversidad (INBio). (2013). Biodiversidad de Costa Rica. Recuperado de: http://www.inbio.ac.cr/ conservacion.html

Kassas, M. (2002). Environmental education: biodiversity. Environmentalist, 22 (4), 345-351.
Leandro, H. (2012, noviembre 15). Juegos ya tienen jefe de misión y su mascota. La Nación. Recuperado de: http:// www.nacion.com/deportes/Juegos-jefe-misionmascota_0_1305469584.html

Riley, S. (2006). Spatial ecology of bobcats and gray foxes in urban and rural zones of a National Park. Journal of Wildlife Management, 70 (5), 1425-1435.

Rosales, L. (2012). Plan de ordenamiento territorial de la Gran Área Metropolitana 2011-2030. San José, Costa Rica. Instituto de Vivienda y Urbanismo.

Sada, M. (2015). The curious case of Costa Rica. Can an outlier sustain its success? Harvard International Review, 36 (4), 11-12.

Salazar, H. (2009). Conservación del patrimonio natural: El caso de la Reserva Biológica Alberto Manuel Brenes. Reflexiones, 88 (1), 77-87.

Sánchez, R. (2006). De caza y cazadores. Las construcciones teóricas sobre la actividad cinegética actual a partir de los discursos de sus actores. Gazeta de Antropología, 22 artículo 18. Recuperado de: http://hdl.handle. net/10481/7100.

Solano, E. (2006). La evolución de la educación ambiental en Costa Rica. Revista de Ciencias Sociales, 111-112 (I), 71-80.

Valerio, R. (1978). Nombres vulgares en la fauna costarricense. San José, Costa Rica. Imprenta Nacional. 
